\title{
Acupuncture: theatrical placebo or caring approach to pain?
}

\author{
Fiona Godlee editor in chief
}

The BMJ

How good is the evidence for acupuncture in managing pain? In our Head to Head this week (doi:10.1136/bmj.k970) the authors on both sides agree that studies show a small but statistically significant effect when it is compared with sham acupuncture. But there they part company. Asbjørn Hróbjartsson and Edzard Ernst say that there is no clinically relevant effect in the randomised trials and that what difference there is can't be distinguished from bias. The fact that studies don't blind the acupuncturists is a fatal flaw, they say. But, says Mike Cummings, sham acupuncture has higher response rates than oral placebo, so randomised controlled trials that use it as the comparator will tend to underestimate acupuncture's effects. He prefers studies that compare acupuncture with usual care.

At issue is whether acupuncture is merely a "theatrical placebo." If this were the basis for its endorsement, argue Hróbjartsson and Ernst, "we should be discussing the ethics of placebo interventions not the elusive effect of acupuncture." Cummings seems to concede this point. "Whether or not you consider these [improvements] to be the effects of a theatrical placebo," he says, "they represent important improvements in quality of life over usual care, and with minimal risk."

The risk and cost, when compared with drug treatments, are further issues of contention. But Kumari Manickasamy took these into account when making her decision to try acupuncture, having exhausted all routes offered by conventional medicine (doi:10.1136/bmj.k990). Struggling with severe pelvic girdle pain during pregnancy, and unable to take higher doses of painkillers, acupuncture offered her hope, she says. It didn't greatly improve the pain, but she found the treatments relaxing. Most importantly, she felt cared for. "It was therapeutic to see an empathetic professional on a regular basis who had the time to listen, who understood my pain, and who was trying to relieve it." Her story suggests that "theatrical placebo" is too dismissive a term for this caring approach to someone in pain. Whether the needles are necessary remains debatable.

Elsewhere this week we explore the links between fluoroquinolones and aortic aneurysm. Björn Pasternak and colleagues confirm an association, suggesting 82 extra cases of aortic aneurysm or dissection within 60 days for every million treatment episodes (doi:10.1136/bmj.k678). The authors point out that this very small increase in absolute risk should be seen in the context of the widespread use of the drugs. Is the association causative? In the linked editorial (doi:10.1136/bmj. k988) David Juurlink walks us through Bradford Hill's nine criteria (listed in case you need reminding) and finds that this association meets most of them. But the survival curves indicate a different explanation: ascertainment bias. Patients who take fluoroquinolones are more likely to have abdominal imaging, as fluoroquinolones are often used in complex urinary tract infections. Nevertheless, the advice remains the same. Prescribe antibiotics judiciously. 